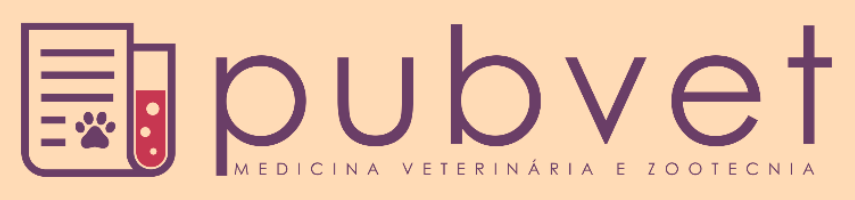

https://doi.org/10.31533/pubvet.v14n8a636.1-6

\title{
Frequência de helmintos em amostras fecais de cães em praças públicas de Pelotas-RS
}

\author{
Tatiana de Avila Antunes ${ }^{1 *} \bullet$, Maysa Seibert de Leão ${ }^{2} \bullet$, Júlia Lignon ${ }^{3} \bullet \mathbb{E}$, João Luis Trojan \\ Camassola $^{2}{ }^{\circ}$, Nicole Freitas Gonçalves ${ }^{\circ} \odot$ (D), Pamela Cristina Andrioli Machado ${ }^{2}$, Alexsander

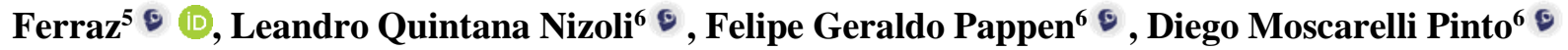 \\ ${ }^{I}$ Doutor em Ciências. Grupo de Estudos em Enfermidades Parasitárias, Universidade Federal de Pelotas, Pelotas-RS Brasil. \\ ${ }^{2}$ Graduando em Medicina Veterinária, Grupo de Estudos em Enfermidades Parasitárias, Universidade Federal de Pelotas, Pelotas- RS Brasil. \\ ${ }^{3}$ Mestranda em Parasitologia Veterinária, Departamento de Microbiologia e Parasitologia, Universidade Federal de Santa Maria-RS Brasil. \\ ${ }^{4}$ Graduanda em Zootecnia, Grupo de Estudos em Enfermidade Parasitarias, Universidade Federal de Pelotas, Pelotas-RS Brasil. \\ ${ }^{5}$ Doutorando, Clínica de Pequenos Animais, Pós-Graduação em Veterinária, Universidade Federal de Pelotas, Pelotas-RS Brasil \\ ${ }^{6}$ Professor da Universidade Federal de Pelotas, Departamento de Veterinária Preventiva Pelotas-RS Brasil. \\ *Autor para correspondência, E-mail: tatdavila@bol.com.br
}

\begin{abstract}
Resumo. O objetivo do presente estudo foi avaliar a frequência de helmintos gastrintestinais de pequenos animais em praças públicas do município de Pelotas-RS. Durante o período de 12 meses do estudo, de outubro de 2018 a setembro de 2019, foram coletadas 470 amostras fecais ambientais com auxílio de sacos plásticos em 10 praças públicas e encaminhadas em recipiente isotérmico para o Laboratório do Grupo de Estudos em Enfermidades Parasitárias (GEEP), FAVET-UFPel, as quais foram processadas utilizando as técnicas coproparasitológicas Willis Mollay (1921) e Sedimentação espontânea (HPJ 1934). Os resultados obtidos demonstraram que 41,1\% (n=193) das amostras foram positivas para algum gênero parasitário, sendo que o helminto de maior ocorrência foi o Ancylostoma spp (57,0\%), seguido por Dipylidium caninum (26,9\%), Trichuris vulpis (10,9\%), Toxocara spp. (4,2\%), Capillaria spp. (0,52\%) e Spirometra spp. $(0,52 \%)$. Conclui-se com a realização deste trabalho que existe a presença de ovos de helmintos com potencial zoonótico em praças públicas da cidade de Pelotas, demonstrando um risco de transmissão de zoonoses para a população que frequenta esses locais, assim como a transmissão entre animais.
\end{abstract}

Palavras chave: fezes, animais, parasitos, zoonose

\section{Helminth frequency in fecal samples from dogs in Pelotas's city public parks}

Abstract. The aim of this present study was to evaluate the frequency of gastrointestinal helminths of small animals in the public parks at Pelotas-RS. During the 12 month period of the study, from October 2018 to September 2019, 470 environmental feces samples were collected with the help of plastic bags in 10 public parks and they were sent in an isothermal container to the Group Study Laboratory on Parasitic Diseases (GEEP), FAVET-UFPel, in which the samples were processed using the Willis Mollay's coproparasitological techniques (1921) and Spontaneous sedimentation (HPJ 1934). The results obtained showed that $41.1 \%(n=193)$ of the samples were positive for some parasitic genus, with the most common helminth being Ancylostoma spp (57.0\%), followed by Dipylidium caninum (26.9\%), Trichuris vulpis (10.9\%), Toxocara spp. (4.2\%), Capillaria spp. $(0.52 \%)$ and Spirometra spp. (0.52\%). It is concluded with the accomplishment of the work that there are helminth eggs whit zoonotic potential in public parks at Pelotas City - RS, 
showing a risk of zoonosis transmission to the population that pass by these places, as well as the transmission among other animals.

Keywords: animals, feces, parasites, zoonosis

\section{Frecuencia de helmintos em muestras fecales en plazas públicas en el municipio de Pelotas-RS}

Resumen. El objetivo del presente estudio fue evaluar la frecuencia de helmintos gastrointestinales de pequeños animales en las plazas públicas de la ciudad de Pelotas-RS. Durante el período de 12 meses del estudio, de octubre de 2018 a septiembre de 2019, se recolectaron 470 muestras fecales ambientales con ayuda de bolsas de plástico en 10 plazas públicas y se enviaron en un contenedor isotérmico al Laboratorio del Grupo de Estudio sobre Enfermedades Parasitarias (GEEP), FAVET-UFPel, que se procesaron utilizando técnicas coproparasitológicas de Willis Mollay (1921) y sedimentación espontánea (HPJ 1934). Los resultados obtenidos mostraron que $41,1 \%(n=193)$ de las muestras fueron positivas para algún género parasitario, siendo el helminto más común Ancylostoma spp (57,0\%), seguido de Dipylidium caninum (26,9\%), Trichuris vulpis (10,88\%), Toxocara spp. (4,2\%), Capillaria spp. (0,52\%) y Spirometra spp. (0,52\%). Con la realización de este trabajo se concluye que existe la presencia de huevos de helmintos con potencial zoonótico en las plazas públicas de la ciudad de Pelotas, lo que demuestra un riesgo de transmisión de zoonosis a la población que frecuenta estos lugares, así como la transmisión entre animales.

Palabras clave: heces, animales, parásitos, zoonosis

\section{Introdução}

Os animais de companhia são cada vez mais importantes na sociedade mundial. Nos últimos anos os pets são como membros da família, contribuindo para o desenvolvimento social, emocional e físico de tutores, crianças e idosos (Campos Filho et al., 2008). Estes animais podem transmitir mais de 60 doenças de caráter zoonótico, entre elas, várias causadas por helmintos e protozoários, sendo considerado um problema de saúde pública a nível mundial, se não for realizado um bom controle e profilaxia dessas enfermidades (Katagiri \& Oliveira-Sequeira, 2007). Tendo em vista à possibilidade de transmissão dessas zoonoses, diversos inquéritos realizados em relação aos geohelmintos demonstram grande relevância das praças públicas como locais que propiciam a transmissão desses parasitos, podendo permanecer no solo durante anos (Araújo et al., 1999; Capuano \& Rocha, 2006; Ferreira et al., 2013; Guimarães et al., 2005; Ross et al., 2011).

A Larva Migrans Visceral (LMV) é caracterizada pela migração do estágio larval de Toxocara spp. e se alojam em diversos órgãos de seres humanos causando processos patológicos hipereosinofílicos crônicos, que podem ser acompanhados por leucocitose e lesões granulomatosas, assim como lesão ocular (LMO) podendo levar a cegueira unilateral. A Larva Migrans Cutânea (LMC) é uma dermatite provocada pela penetração ativa em pele integra e migração no estrato epitelial em humanos, sendo que no Brasil, Ancylostoma braziliense e A. caninum, constituem os principais nematódeos envolvidos. Outros helmintos importantes são o Trichuris spp. e Dipylidium sp (Evaristo et al., 2019; Ferreira et al., 2013; Guimarães et al., 2005; Katagiri \& Oliveira-Sequeira, 2007; Santarém et al., 2002; Taylor et al., 2017). O objetivo do presente estudo foi avaliar a frequência de helmintos em fezes do ambiente de cães nas principais Praças Públicas da cidade de Pelotas, Rio Grande do Sul-Brasil no período de 12 meses.

\section{Material e métodos}

As coletas foram realizadas em 10 praças públicas no município de Pelotas-RS, que apresenta as seguintes características: latitude sul $31^{0} 46^{`} 19^{\prime}$ e longitude ocidental $52^{\circ} 20^{\prime} 33^{\prime \prime}$, população de 328 . 275 habitantes, segundo o censo do IBGE (2010), distribuídos em uma área de $1.609 .708 \mathrm{~km}^{2}$. Situa-se na região Sul do Rio Grande do Sul fazendo parte do bioma pampa e possui clima subtropical úmido e temperado. O critério de escolha das praças baseou-se no fato de apresentarem maior circulação de 
pessoas e abrangerem zonas centrais e periféricas da cidade, sendo estipulado como pontos de coleta quatro laterais externos e um ponto central, selecionando-se as não ressecadas e com ausência de crescimento de fungos. As amostras fecais foram coletadas das seguintes praças: Praça Coronel Pedro Osório, Praça do Colono, Praça do Terminal Rodoviário, Praça Dom Joaquim, Praça Dom Antônio Zattera, Praça Piratinino de Almeida, Praça Cipriano Barcelos, Praça Domingos Rodrigues, Praça Juscelino Kubitschek com Gonçalves Chaves e Praça Lorival Ângelo.

As amostras fecais foram coletadas mensalmente no período de outubro de 2018 a setembro de 2019, totalizando 470 amostras, sendo todas coletadas individualmente com auxílio de sacos plásticos e acondicionadas em caixas isotérmicas contendo gelo biológico e enviadas ao laboratório. As análises foram realizadas no Laboratório do Grupo de Estudos em Enfermidades Parasitárias (GEEP), pertencente à Faculdade de Veterinária da Universidade Federal de Pelotas.

As técnicas coproparasitológicas utilizadas para a pesquisa de ovos de helmintos nas amostras de fezes foram: a Técnica de Willis-Mollay (1921) que tem como princípio a flutuação. A outra técnica utilizada foi a de Sedimentação Espontânea em água (HPJ) descrita por Hoffman et al. (1934) que é utilizada para pesquisa de ovos pelo princípio da sedimentação.

\section{Resultados}

Do total de 470 amostras analisadas, 193 foram positivas para algum gênero de parasito gastrintestinal, representando uma frequência anual de 39,8\%. Das amostras positivas $(n=193)$, o helminto de maior frequência foi o Ancylostoma spp, 110/193 (57,0\%), seguido por Dipylidium caninum 52/ 193 (26,9\%), Trichuris vulpis 21/193 (10,88\%), Toxocara spp. 8/193 (4,15\%), Capillaria spp. 1/193 $(0,52 \%)$ e Spirometra spp. 1/193 (0,52\%). Os resultados estão descritos na Tabela 1. As frequências de ovos de helmintos encontrados mensalmente nas 10 praças coletadas estão dispostas na Tabela 2 e Figura 1.

Tabela 1. Frequência de parasitos encontrados em amostras fecais de ambiente em praças públicas no município de PelotasRS no período de outubro de 2018 a setembro de 2019

\begin{tabular}{lccc}
\hline Gênero parasitário & $\begin{array}{c}\text { Amostras positivas } \\
(\mathrm{n} / 193)\end{array}$ & $\begin{array}{c}\text { Frequência (\%) em relação aos Frequência (\%) em relação ao } \\
\text { positivos }(\mathrm{n}=193)\end{array}$ & $\begin{array}{c}\text { total amostras }(\mathrm{n}=470) \\
\text { Ancylostoma } \text { spp. }\end{array}$ \\
Dipylidium caninum & $110 / 193$ & 56,99 & 11,06 \\
Trichuris vulpis & $52 / 193$ & 26,94 & 4,46 \\
Toxocara spp. & $21 / 193$ & 10,88 & 1,70 \\
Capillaria spp. & $8 / 193$ & 4,15 & 0,21 \\
Spirometra spp. & $1 / 193$ & 0,52 & 0,21 \\
\hline
\end{tabular}

Fonte: GEEP.

Tabela 2. Frequência relativa (\%) mensal de ovos de helmintos em amostras fecais do ambiente coletadas em 10 praças públicas no município de Pelotas-RS

\begin{tabular}{lccc}
\hline Meses/anos & $\begin{array}{c}\text { Número de amostras } \\
\text { coletadas }\end{array}$ & $\begin{array}{c}\text { Número de amostras } \\
\text { positivas }\end{array}$ & Frequência mensal, \% \\
\hline Outubro/2018 & 36 & 21 & 58,33 \\
Novembro/2018 & 50 & 22 & 44 \\
Dezembro/2018 & 46 & 14 & 30,43 \\
Janeiro/2019 & 26 & 13 & 50 \\
Fevereiro/2019 & 35 & 11 & 31,43 \\
Março/2019 & 32 & 15 & 46,88 \\
Abril/2019 & 46 & 22 & 47,83 \\
Maio/2019 & 42 & 16 & 38,10 \\
Junho/2019 & 35 & 14 & 40 \\
Julho/2019 & 37 & 15 & 40,54 \\
Agosto/2019 & 49 & 19 & 38,78 \\
Setembro/2019 & 36 & 11 & 30,56 \\
\hline Total & 470 & 187 & 41,06 \\
\hline Fonte: GEEP
\end{tabular}

Fonte: GEEP. 


\section{Discussão}

Do total de amostras analisadas (470), 41,1\% estavam parasitadas com ovos de helmintos gastrintestinais, estes resultados não foram semelhantes aos encontrados por Alves et al. (2014) que coletaram amostras fecais de cães de oito praças públicas de Pindamonhangaba-SP durante período de setembro de 2010 a setembro de 2011 e das 640 amostras coletadas $27,2 \%$ foram positivos para algum gênero parasitário; porém, corroboraram com o estudo de Almeida et al. (2007) que tiveram 37,19\% de amostras positivas de um total de 121 amostras coletadas em Cuiabá, MT. Os resultados do presente estudo também corroboraram com estudos de Ross et al. (2011) que obtiveram $34 \%$ de positividade das 100 amostras analisadas em 10 praças de Cruz Alta, RS no período de Junho (outono). Entretanto, os resultados do estudo diferiram dos encontrados por Blazius et al. (2005) que obtiveram 76,6\% das amostras positivas das 158 analisadas em Itapema, SC, possivelmente por terem sido coletadas durante 12 meses somente de cães errantes apreendidos pela vigilância epidemiológica do município.

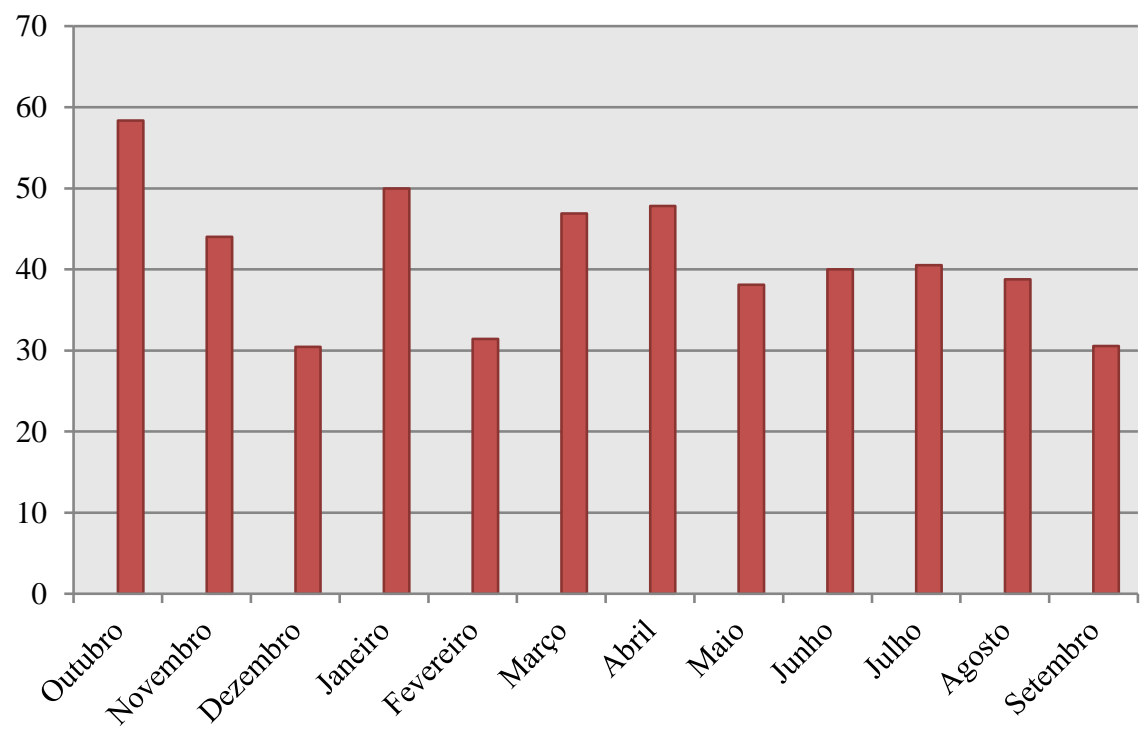

Figura 1. Frequência (\%) mensal de ovos de helmintos em amostras fecais do ambiente coletadas em 10 praças públicas no município de Pelotas-RS.

Em resultados obtidos por Campos Filho et al. (2008) analisando 119 amostras fecais de cães e gatos de dez praças públicas da área urbana do município de Itabuna-Bahia, em um ano de estudo foram diagnosticadas 47,9\% de amostras contaminadas por ovos de Ancylostoma spp., valor inferior ao encontrado no presente trabalho que foi de 57,0\%; porém, em ambos os estudos foi o parasito de maior prevalência. Outros trabalhos, como os de Alves et al. (2014) e de Pedrassani et al. (2008) em Canoinhas, $\mathrm{SC}$, também demonstraram que o Ancylostoma spp. foi o parasito de maior frequência nas amostras analisadas, com isso deve-se levar em conta a relevância desse helminto como um risco de potencial zoonótico por ser o causador da Larva Migrans Cutânea (LMC) nos seres humanos (Ferreira et al., 2013). Outro estudo realizado por Evaristo et al. (2019) na cidade de Pedro Osório, RS, em duas praças públicas as quais foram coletadas amostras de fezes ambientais de cães no período de dezembro de 2017 e novembro de 2018, demostrou que 61,1\% das amostras eram positivas para Ancylostoma spp. , corroborando com o presente trabalho. A maior frequência desse parasito nas amostras analisadas pode ser explicada pelo fato de caninos e felinos se contaminarem via trans mamária e transplacentária, ingestão e penetração ativa de larvas em estágio de L3 (Chandler et al., 2006).

No presente estudo outros parasitos com potencial zoonótico encontrados foram o Dipylidium caninum, Trichuris vulpis e Toxocara spp., sendo este último o responsável pela Síndrome da Larva Migrans Visceral (LMV) e Larva Migrans Ocular (LMO) em humanos. Estes resultados corroboram com outros trabalhos realizados em locais públicos que também encontraram ovos desses parasitos nas fezes de cães (Alves et al., 2014; Pedrassani et al., 2008). Em relação ao Toxocara spp. a frequência encontrada foi de 4,2\%, resultado semelhante ao encontrado por Campos Filho et al. (2008) a qual foi de 4,2\% em um estudo em 10 praças públicas no município de Itabuna-BA, porém não corrobora com 
estudo realizado por Blazius et al. (2005) que apresentaram frequência de 14,5\% das amostras positivas para Toxocara spp nas fezes coletadas de cães errantes em Itapema, SC, nem com os resultados obtidos por Pedrassani et al. (2008), o qual não encontrou ovos de Toxocara spp nas fezes cães em área de lazer da cidade de Canoinhas, SC.

No presente estudo foram encontrados somente um ovo de Spirometra spp. $(0,52 \%)$ e um ovo de Capillaria spp. (0,53\%); porém, em outros trabalhos realizados em praças públicas não identificaram ovos deste parasito (Alves et al., 2014; Campos Filho et al., 2008; Pedrassani et al., 2008).

As técnicas coproparasitológicas realizadas no presente estudo, que foram Willis-Mollay (1921) e Sedimentação Espontânea (HPJ, 1934) foram às mesmas realizadas em outros estudos demonstrando serem eficazes para o diagnóstico parasitológico, tanto para ovos leves com câmara de ar e ovos pesados como o do Dipylidium caninum, respectivamente (Alves et al., 2014; Capuano \& Rocha, 2006; Ferraz et al., 2019; Kepps et al., 2006).

Existe divergência quanto à prevalência de helmintos em diferentes partes do mundo, devido a diferentes condições ambientais, climáticas e epidemiológicas, que podem estar ligadas a fatores socioeconômicos e culturais de cada região (Bojanich et al., 2015). De acordo com o estudo de Alves et al. (2014) as estações do ano de maior prevalência de ovos de parasitos em fezes de cães foram no início do verão e no outono. Este estudo foi realizado na cidade de Pindamonhangaba-SP, que apresenta temperaturas diferentes das encontradas no RS, com as estações do ano não apresentando tantas diferenças climáticas. Entretanto, no presente trabalho as maiores frequências ocorreram nos meses de outubro, novembro, janeiro, abril e maio, ou seja, na primavera, início do verão e outono, com menor percentual no inverno. A diminuição da frequência de parasitos no inverno pode ser explicada por ser normalmente meses de frio intenso e períodos de muita chuva, como no estudo de (Pedrassani et al., 2008) que não apresentou nenhuma amostra positiva em julho.

\section{Conclusão}

Conclui-se com a realização deste trabalho que existe a presença de ovos de helmintos com potencial zoonótico em praças públicas da cidade de Pelotas, demonstrando um risco de transmissão de zoonoses para a população que frequenta esses locais, assim como a transmissão entre animais.

\section{Referências bibliográficas}

Almeida, A. B. P., Sousa, V. R. F., Dalcin, L., \& Silva Justino, C. H. (2007). Contaminação por fezes caninas das praças públicas de Cuiabá, Mato Grosso. Brazilian Journal of Veterinary Research and Animal Science, 44(2), 132-136. DOI: https://doi.org/10.11606/issn.1678-4456.bjvras.2007.26651

Alves, A. P. S. M., Coelho, F. A. S., \& Coêlho, M. D. G. (2014). Frequência De Enteroparasitos Em Fezes De Cães Coletadas Em Praças Públicas Do Município De Pindamonhangaba, SP, Brasil. Revista de Patologia Tropical, 43, 341-350. DOI: https://doi.org/10.5216/rpt.v43i3.32204

Araújo, F. R., Crocci, A. J., Rodrigues, R. G. C., Silva Avalhaes, J., Miyoshi, M. I., Salgado, F. P., Silva, M. A., \& Pereira, M. L. (1999). Contaminação de praças públicas de Campo Grande, Mato Grosso do Sul, Brasil, por ovos de Toxocara e Ancylostomaem fezes de cães. Revista Da Sociedade Brasileira de Medicina Tropical, 32(5), 581-583. DOI: https://doi.org/10.1590/s003786821999000500017

Blazius, R. D., Emerick, S., Prophiro, J. S., Romão, P. R. T., \& Silva, O. S. (2005). Ocorrência de protozoários e helmintos em amostras de fezes de cães errantes da cidade de Itapema, Santa Catarina. Revista Da Sociedade Brasileira de Medicina Tropical, 38(1), 73-74. DOI: https://doi.org/10.1590/s0037-86822005000100018

Bojanich, M. V., Alonso, J. M., Caraballo, N. A., Itatí Schöller, M., López, M. de los Á., García, L. M., \& Basualdo, J. Á. (2015). Assessment of the presence of Toxocara eggs in soils of an arid area in central-western Argentina. Revista Do Instituto de Medicina Tropical de São Paulo, 57(1), 73-76. DOI: https://doi.org/10.1590/s0036-46652015000100010

Campos Filho, P. C., Barros, L. M., Campos, J. O., Braga, V. B., Cazorla, I. M., Albuquerque, G. R., \& Carvalho, S. M. S. (2008). Parasitas zoonóticos em fezes de cães em praças públicas do município 
de Itabuna, Bahia, Brasil. Revista Brasileira de Parasitologia e Veterinária, 17(4), 206-209. DOI: https://doi.org/10.1590/s1984-29612008000400007

Capuano, D. M., \& Rocha, G. M. (2006). Ocorrência de parasitas com potencial zoonótico em fezes de cães coletadas em áreas públicas do município de Ribeirão Preto, SP, Brasil. Revista Brasileira de Epidemiologia, 9(1), 81-86. DOI: https://doi.org/10.1590/s1415-790x2006000100010

Chandler, E. A., Gaskell, C. J., \& Gaskell, R. M. (2006). Clínica e terapêutica em felinos. Editora Roca.

Evaristo, T. A., Lignon, J. S., Pellegrin, T. G., Camassola, J. L., Ferraz, A., Leão, M. S., Fuentes, G. G., Antunes, T. Á., Pappen, F. G., \& Pinto, D. M. (2019). Frequência de Ancylostoma spp. em amostras fecais ambientais nas praças públicas de Pedro Osório, RS. PUBVET, 14(4), 1-7. DOI: https://doi.org/10.31533/pubvet.v13n4a315.1-7

Ferraz, A., Evaristo, T. A., Coelho, A. L. R., Castro, T. A., Mello, C. C. S., Pappen, F. G., Silva, S. S., \& Nizoli, L. Q. (2019). Presença de parasitos com potencial zoonótico na areia de praças de recreação de escolas municipais de educação infantil do município de Pelotas, RS, Brasil. Veterinária e Zootecnia, 26, 1-7. DOI: https://doi.org/10.35172/rvz.2019.v26.147

Ferreira, F. P., Dias, R. C. F., Martins, T. A., Constantino, C., Pasquali, A. K. S., Vidotto, O., Freire, R. L., \& Navarro, I. T. (2013). Frequência de parasitas gastrointestinais em cães e gatos do município de Londrina, PR, com enfoque em saúde pública. Semina: Ciências Agrárias, 34(6), 3851-3858. DOI: https://doi.org/10.5433/1679-0359.2013v34n6supl2p3851

Guimarães, A. M. ., Alves, E. G. L., \& Rezende, G. F. (2005). Ovos de Toxocara sp. e larvas de Ancylostoma sp. em praça pública de Lavras, MG. Revista de Saúde Pública, 39(2), 293-295. DOI: https://doi.org/10.1590/s0034-89102005000200022

Hoffman, W. A., Pons, J. A., \& Janer, J. L. (1934). The sedimentation-concentration method in schistosomiasis mansoni. 283-291.

IBGE- Instituto Brasileiro de Geografia e Estatística. Pesquisa Nacional de Saúde. (2013). Acesso em 02 de fevereiro de 2020

Katagiri, S., \& Oliveira-Sequeira, T. C. G. (2007). Zoonoses causadas por parasitas intestinais de cães e o problema do diagnóstico. Arquivos Do Instituto Biológico, 74(2), 175-184.

Kepps, M., Dionello, M. A., Gatti, F., Susin, L. R. O., Signorini, V. R. M., \& Scaini, C. J. (2006). Infecção por parasitas, com potencial zoonótico, em cães semidomiciliados e domiciliados. XIX Congresso Brasil itologia.eiro de Paras

Pedrassani, D., Viera, A. M., \& Thiem, E. M. B. (2008). Contaminação por Toxocara spp. e Ancylostoma spp. em áreas de lazer do município de Canoinhas, SC. Archives of Veterinary Science, 13(2), 110-117. DOI: https://doi.org/10.5380/avs.v13i2.12895

Ross M., Schmitt, B.A.M, De-Paula D., Tomazzi, R.C, Cecchin, R.S., Kunh F., Felippin T., Sperotto, R.L., Zanella, J.F.P. (2011). Prevalência de ovos, larvas, cistos e oocistos de parasitas com potencial zoonótico em praças públicas e áreas de lazer na cidade de Cruz Alta, RS: Análise preliminar. Anais: XVI Seminário interinstitucional de ensino, pesquisa e extensão.

Santarém, V. A., Andrade, S. F., \& Alberti, H. (2002). Endoparasiticidas e ectoparasiticidas. Manual de Terapêutica Veterinária, 2, 437-476.

Taylor, M. A., Coop, R. L., \& Wall, R. L. (2017). Parasitologia Veterinária. Guanabara Koogan.

Willis-Mollay, H. H. (1921). A simple levitation method for the detection of hookworm ova. Medical Journal of Australia, 2(18), 375-376. DOI: https://doi.org/10.5694/j.1326-5377.1921.tb60654.X

Recebido: 10 de março, 2020.

Aprovado: 6 de abril, 2020

Disponível online: 27 de agosto, 2020.

Licenciamento: Este artigo é publicado na modalidade Acesso Aberto sob a licença Creative Commons Atribuição 4.0 (CC-BY 4.0), a qual permite uso irrestrito, distribuição, reprodução em qualquer meio, desde que o autor e a fonte sejam devidamente creditados. 